\title{
Effects of the hospital-community-family ternary linkage continuous nursing model on compliance, cognitive function, resilience, and quality of life for children with epilepsy: a retrospective study
}

\author{
Yali Gao ${ }^{1 \#}$, Xiaohong Tang ${ }^{1 \#}$, Yiyi Wen ${ }^{1}$, Daijing Qian ${ }^{1}$, Xingnan Pan ${ }^{2}$, Linhui Zhang ${ }^{1}$ \\ ${ }^{1}$ Department of Neurology, Wuxi Children's Hospital, Wuxi, China; ${ }^{2}$ Department of Science and Education, Wuxi Children's Hospital, Wuxi, China \\ Contributions: (I) Conception and design: Y Gao, X Tang; (II) Administrative support: X Pan, L Zhang; (III) Provision of study materials or patients: \\ Y Wen, D Qian; (IV) Collection and assembly of data: Y Gao; (V) Data analysis and interpretation: All authors; (VI) Manuscript writing: All authors; \\ (VII) Final approval of manuscript: All authors. \\ \#These authors contributed equally to this work. \\ Correspondence to: Linhui Zhang. Department of Neurology, Wuxi Children's Hospital, No. 299, Qingyang Road, Liangxi District, Wuxi 214000, \\ China. Email: $372141285 @ q q . c o m ;$ Xingnan Pan. Department of Science and Education, Wuxi Children's Hospital, No. 299, Qingyang Road, \\ Liangxi District, Wuxi 214000, China. Email: 2902466433@qq.com.
}

\begin{abstract}
Background: Epilepsy is a chronic disease that can reduce the quality of life in children because it can cause memory loss, learning difficulties, mental and behavioral abnormalities, and social disorders. The purpose of this study was to explore the effect of the hospital-community-family linkage continuous nursing model in the nursing of children with epilepsy.

Methods: Data for 136 children with epilepsy who were hospitalized in the neurology clinic or neurology ward of Wuxi Children's Hospital from January 2018 to January 2021 were retrospectively analyzed. According to the nursing mode after discharge, the children were divided into 65 cases in the observation group (hospital-community-family linkage continuous nursing model) and 71 cases in the control group (routine nursing model). The compliance with nursing, cognitive function, resilience, and quality of life of the two groups were compared before and after intervention, and the influencing factors of children's quality of life were analyzed.
\end{abstract}

Results: The follow-up visit 6 months after discharge found that the scores for compliance, resilience, and quality of life in the observation group were significantly higher than those in the control group $(\mathrm{P}<0.05)$. The correlation analysis between quality of life, each child's general data, and the nursing model found that the total score of quality of life was correlated with incidence frequency, family per capita income, and the nursing model.

Conclusions: The adoption of a hospital-community-family linkage continuous nursing model can improve the compliance, resilience, and quality of life of children with epilepsy.

Keywords: Epilepsy; hospital-community-family; quality of life; compliance; resiliency; cognitive function

Submitted Dec 22, 2021. Accepted for publication Feb 11, 2022.

doi: $10.21037 / \mathrm{tp}-22-21$

View this article at: https://dx.doi.org/10.21037/tp-22-21

\section{Introduction}

Epilepsy (EP) is a chronic brain disease that is common in children and is characterized by repeated abnormal brain discharges that have various causes. The health status of children with EP is affected by many factors, such as onset age, course of disease, seizure type, seizure frequency and 
so on. According to the statistics of 2017 , there are about 10.5 million active epileptic children in the world, $80 \%$ of which live in developing countries and economically underdeveloped countries (1). The prevalence of EP in children in China is $5-10 \%$ (2); there are about 6 million children with active EP in China, and 400,000 new cases occur every year (3). The indirect impacts of EP on children and families far exceeds the direct impact of EP itself, and EP has become a public issue of global concern (4). The repeated onset of EP not only affects the physical and mental health of children, but also may lead to sudden death. Many studies have shown that children with EP have a low quality of life, mainly as a result of memory loss, learning difficulties, mental and behavioral abnormalities, and social disorders (5). Due to the unpredictability of the onset of EP and the poor self-protection ability of children, there are many potential safety hazards. Due to the lack of public understanding and about EP, the physical appearance of EP in children is often a target of discrimination by others and can result in a strong sense of shame for children and caregivers (6). EP also increases the economic burden of family and society. Compared with asthma, diabetes, and other chronic diseases, children with EP have the highest family annual medical expenditure of up to $\$ 9,103.25$ (7). The main treatment of EP is antiepileptic drug (AED). EP in between $70 \%$ and $80 \%$ of children can be effectively controlled by long-term medication, but between $12 \%$ and $35 \%$ of children have poor treatment compliance (8). Children's poor compliance with treatment can lead to repeated EP attacks, drug resistance, and an increase in the number of hospitalizations, which affect the quality of life of children with EP (9). Educating caregivers and providing information support during EP treatment can improve caregivers' disease management ability and treatment compliance and subsequently improve children's health indicators (10).

At present, there is no unified standard for EP management in China and abroad. Some research reports have shown that the nursing of children's EP is best carried out by specialized nurses in communities or hospitals (11). Ternary linkage, namely hospital-community-family linkage continuous nursing, enables a seamless connection between treatment and nursing for patients returning from the hospital to their normal family life, and improve the maximum utilization rate of medical resources. The main medium of ternary linkage continuous nursing is the nursing service participated by the hospital and the community. At present, in China, the ternary linkage extended nursing mode is applied to many chronic diseases such as stroke, coronary heart disease, diabetes mellitus, and renal failure. In 2015, Wuxi Children's Hospital began to carry out management cooperation for children with EP with 10 communities in Wuxi. We gradually expanded the scale and summarized our rich experience in management of children's EP. From January 2018 to January 2021, the Department of Neurology of our hospital implemented the hospital-community-family linkage continuous nursing model for 65 children with EP in 10 communities, with good results. We present the following article in accordance with the STROBE reporting checklist (available at https:// tp.amegroups.com/article/view/10.21037/tp-22-21/rc).

\section{Methods}

\section{Research participants}

This study retrospectively collected the data of 136 children with EP who were hospitalized in the neurology clinic or neurology ward of Wuxi Children's Hospital from January 2018 to January 2021. The study was conducted in accordance with the Declaration of Helsinki (as revised in 2013). The study was approved by the Ethics Committee of Wuxi Children's Hospital (No. 2020451). Informed consent was obtained from all patients' guardians. They met the following inclusion criteria: (I) the child's diagnosis met the diagnostic criteria for EP formulated by the International League Against Epilepsy (ILAE) in 2014 (12); (II) the hospitalization within the study timeframe was the child's first visit to Wuxi Children's Hospital for EP; (III) the child was age $\leq 14$ years with a follow-up time $>6$ months; (IV) we had access to the child's complete medical records; and (V) we obtained informed consent of the child's guardian.

Patients were excluded if they had the following: (I) incomplete clinical data; (II) other nervous system diseases, psychiatric diseases or other physical diseases; (III) nondrug treatment, such as surgical treatment of children with EP; or (IV) abnormal vision or hearing; or (V) the child or their guardian refused to participate in the study.

\section{Nursing model}

\section{Routine nursing model Regular health education}

During hospitalization, patients are given routine health education, they are informed of disease-related knowledge (EP attack, inducement, medication precautions, etc.), and we collect general data, including relevant information 
of children with EP and their parents. Routine discharge guidance is given at the time of discharge, including medication guidance (information about the role of the drugs used, adverse reactions, how to obtain the accurate dose, precautions for taking, etc.), emergency treatment knowledge (such as emergency treatment of convulsions), nutrition (precautions for drinking and eating while taking drugs, etc.), and exercise guidance (such as which sports children with EP can do safely).

\section{Telephone follow-up}

After discharge, a nurse records the telephone number of the department on the discharge record to provide telephone consultation to children with EP and their parents. Every 2 weeks, the nurses who received training in this subject conducted a telephone follow-up call with children with EP and their parents in which they asked about the health status, medication status, and living conditions of the child with EP, and answered the relevant questions raised by the child with EP and their parents in a timely and detailed manner. The telephone follow-up time was not less than 15 minutes long. At the end of the followup, the nurse reminded the child with EP and their parents to take their drugs on time, maintain a peaceful state of mind, and make their return visit on time.

\section{Pay a return visit}

After every 3 months, nurses called the child's parents to inform them that they need to come to the hospital for review in the near future (within 1 week).

\section{Hospital-community-family ternary linkage continuous nursing model}

\section{Establish a networked platform}

The platform includes patient information, a nursing demand survey, remote nursing consultation, hospital community two-way linkage training, health knowledge base, continuous nursing WeChat group, and other modules.

\section{Establish a ternary linkage group}

The team consists of 1 chief physician, 1 head nurse, 6 hospital nurses, 3 community nurses, and 1 parent, guardian, or caregiver. Centralized training shall be conducted before the child with EP is discharged from hospital and will mainly focus on the basic knowledge of $\mathrm{EP}$ and first aid knowledge to clarify the responsibilities of continuous nursing for the hospital, community, and family.

\section{Hospital responsibilities}

The hospital is responsible for: the design and organization of the whole project; sharing some patient information and high-quality nursing teaching resources with the community through the platform; realizing specialized technical guidance; undertaking remote consultation and two-way consultation; and following up and supervising the continuous nursing plan. During the hospitalization of patients, the nursing staff of the ternary linkage group are responsible for: investigating the continuous nursing needs of patients; formulating nursing plans; introducing the use of the platform to patients; and guiding patients to choose nearby communities for services.

Regularly push theoretical knowledge of EP, medication, diet and first-aid knowledge in the form of pictures, videos and texts on the WeChat public account. Ensure at least 2 nurses online in the WeChat group. Parents can consult and respond whenever they have problems. Difficult problems can also contact doctors for remote guidance. The hospital nurse shall count the follow-up time of each child and remind them of their follow-up visit through WeChat or by telephone 3 days in advance.

\section{Community responsibilities}

Community is a residential area formed by the merger of residents' committees. The community is mainly responsible for family visits. Within 6 months after discharge, community nurses conduct home visits every 2 weeks to evaluate the rationality of medication and diet, take the patient's weight, help the patient to understand EP control, answer parents' doubts face to face, and emphasize the importance of medication treatment again.

\section{Family responsibilities}

Caregivers understand the continuous nursing and relevant news through the platform, view the relevant health knowledge pushed by WeChat, and take good care of children.

\section{Observation indexes}

\section{Medication compliance questionnaire}

To check the compliance with treatment of children with EP, we used the Morisky Medical Compliance Scale 4-item Version (13) design and included the following four questions: (I) Have you forgotten to take your EP medicine? (II) Do you sometimes not pay attention to your EP medication? (III) Have you ever stopped taking your EP medicine when you feel your symptoms improve? (IV) Have you ever stopped taking your EP medicine when you feel your symptoms are worse?

To evaluate treatment compliance, if the answer to all four questions is "no", it means good compliance, and if 
more than one of the four questions answers "yes", it means poor compliance.

\section{Cognitive function questionnaire}

Neuropsychological tests were conducted before discharge and 6 months after discharge, with reference to the Wechsler Intelligence Scale for Children (WISC) (14). According to Wechsler's intelligence level: an full intelligence quotient (FIQ) score of below 69 points shows an intelligence defect, $70-79$ points shows that intelligence in the boundary state, 80-89 points shows that intelligence is below the normal level, 90-109 points shows that intelligence is normal, 110-119 points shows that intelligence is above the normal level, 120-129 points shows that intelligence is extraordinary, and more than 130 points shows that intelligence is extremely extraordinary. The highest score of verbal intelligence quotient (VIQ) and performance intelligence quotient (PIQ) was 159 , VIQ and PIQ <70 were intellectual impairment, 70-90 were marginal intellectual state, and >90 were normal.

\section{Resilience questionnaire}

Resilience was measured with an instrument developed by Kim (15). The tool measures the intrapersonal, coping, and interpersonal aspects of children with chronic diseases. The tool has 32 questions on a 4-point scale. Scores range from a minimum of 32 to a maximum of 128 . The higher the score, the stronger the resilience of the child.

\section{Quality of life questionnaire}

The Quality of Life Inventory Measurement Models (PedsQL) compiled by Varni in 1987 and translated by Lu et al. (16) was used to evaluate the quality of life of children with EP. The scale contains 23 items, which are divided into 4 aspects. Among them, the physiological function includes 8 items, the emotional function includes 5 items, the social function includes 5 items, and the role (school performance) function includes 5 items. Each item adopts 5 grades of 0 to 4 to evaluate the frequency of something in the last month, and the score is converted to 0 to 100 points. The score of each aspect is the sum of the scores of subordinate items in the aspect divided by the number of items contained in the aspect. The score of the total table is the sum of the scores of each item divided by the number of items in the whole table. The higher the score, the better the quality of life.

\section{Statistical analysis}

After classifying and numbering the questionnaire, the statistical program of social science software version 22.0 (SPSS Inc., Chicago, IL, USA) was used for statistical analysis. The mean \pm standard deviation (SD) was used for the measurement data of normal distribution, and classified data were expressed in frequency and percentage. The $t$-test of independent samples was used for intergroup comparison. The Chi square test was used to compare frequency data between groups. The statistical significance indexes of univariate analysis were included, and bivariate logistic regression analysis was used in multivariate analysis. $\mathrm{P}<0.05$ was considered statistically significant.

\section{Results}

\section{Basic patient characteristics}

Among the 136 patients with esophageal cancer included in this study, 76 were boys and 60 were girls, aged 4-14 years, with an average age of 6.11 years. There were 65 cases in the observation group and 71 cases in the control group. There was no significant difference in general data between the two groups (see Table 1, Figure 1).

\section{Compliance analysis}

Medication compliance of epileptic children in the observation group increased from $33.8 \%$ before discharge to $76.9 \%$, and medication compliance of the control group increased from $39.4 \%$ before discharge to $46.5 \%$, and there were significant differences between the two groups before and after nursing $(\mathrm{P}<0.05)$. There was a significant difference in compliance between the two groups 6 months after discharge $(\mathrm{P}<0.05$; Table 2$)$.

\section{Resiliency analysis}

The resilience of children with EP in the observation group and the control group increased from $90.26 \pm 15.33$ to $98.75 \pm 8.38$ and $90.73 \pm 12.68$ to $94.81 \pm 9.67$, respectively, after nursing $(\mathrm{P}<0.05)$. Six months after discharge, there was no significant difference in resilience between the two groups ( $>0.05$; Table 2).

\section{Cognitive function}

The scores of VIQ and FIQ of children with EP in the observation group and the control group before discharge and 6 months after discharge were significantly higher than 
Table 1 Baseline characteristics of children with EP

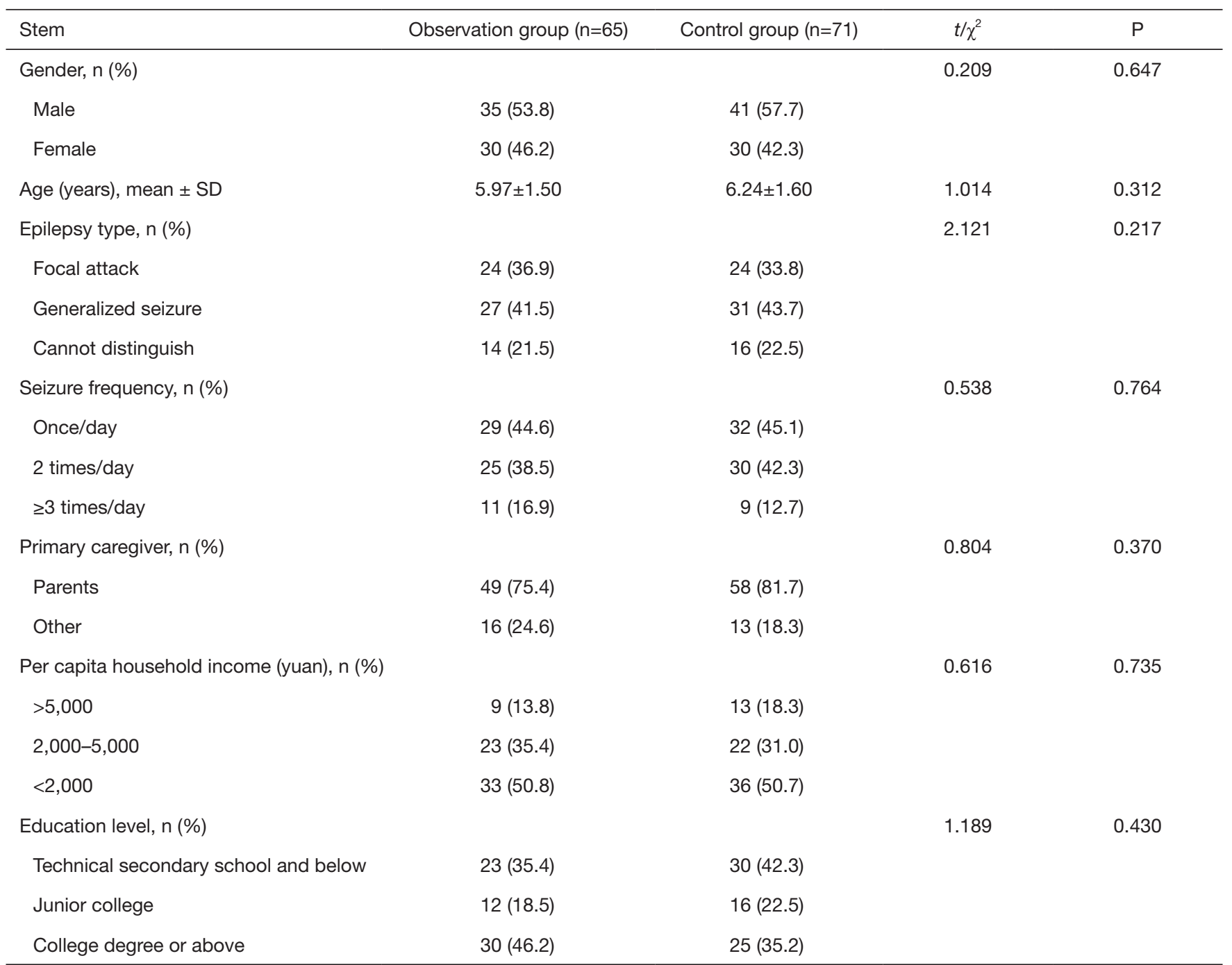

EP, epilepsy; SD, standard deviation.

those before treatment $(\mathrm{P}<0.05$; Table 3).

\section{Quality of life}

The scores of physical function, emotional health, and behavioral function of children with EP in the observation group and the control group before discharge and 6 months after discharge were higher than before the treatment, and the difference was statistically significant $(\mathrm{P}<0.05)$. The improvement of social function was not obvious, and the difference was not statistically significant $(\mathrm{P}>0.05)$. Six months after discharge, there were significant differences in physical function, emotional health, and behavioral function between the two groups $(\mathrm{P}<0.05 ;$ Table 4$)$.

\section{Correlation analysis and regression analysis of quality of life}

The correlation analysis found that the total score of quality of life was correlated with attack frequency, family per capita income, and grouping (Table 5).

To test this relationship further, multiple stepwise regression analyses were carried out with the total score of quality of life as the dependent variable and the meaningful variables of univariate analysis as the independent variable. The results showed that the total score of quality of life was affected by attack frequency, family per capita income, and 


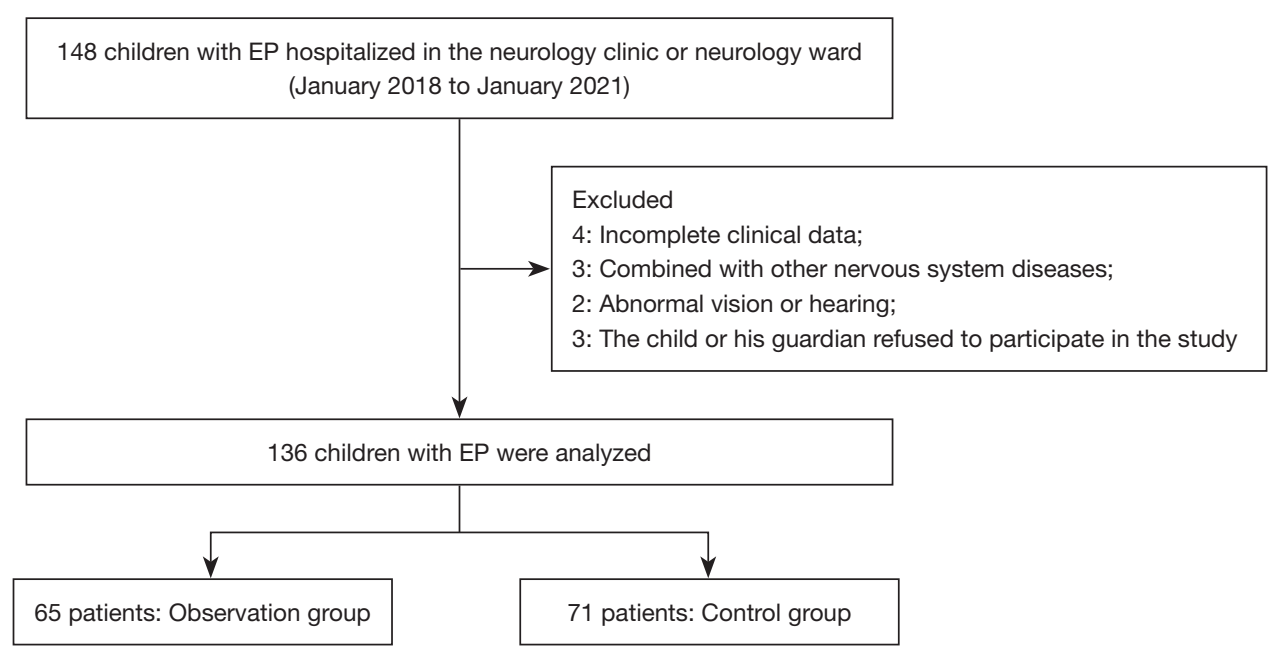

Figure 1 Study flowchart. EP, epilepsy.

Table 2 Compliance and resilience of children with EP

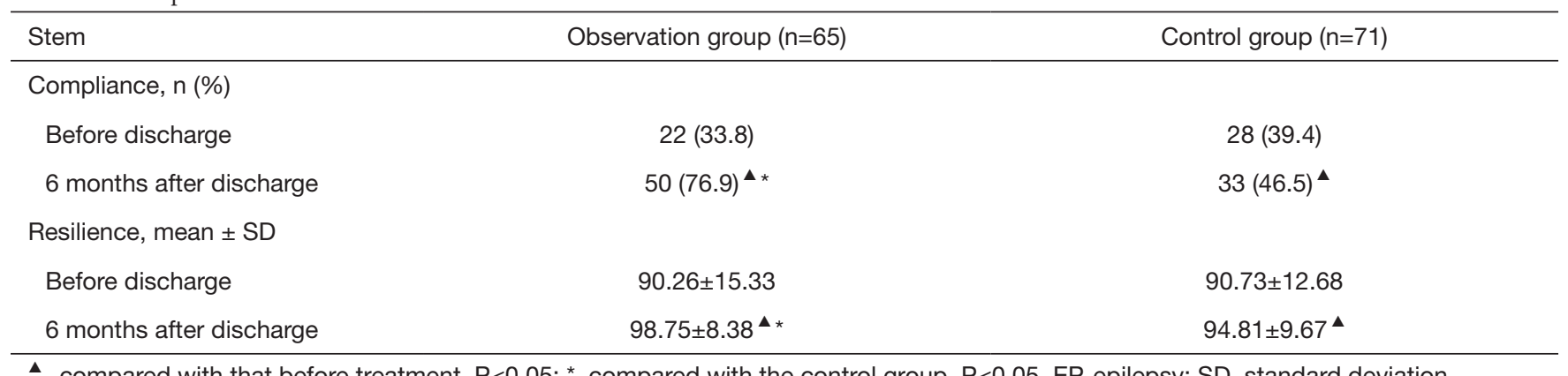

$\triangle$, compared with that before treatment, $\mathrm{P}<0.05$; ${ }^{*}$, compared with the control group, $\mathrm{P}<0.05$. EP, epilepsy; SD, standard deviation.

Table 3 Cognitive function of children with $\mathrm{EP}($ mean $\pm \mathrm{SD})$

\begin{tabular}{lccc}
\hline Group & VIQ & PIQ & FIQ \\
\hline Observation group $(n=65)$ & & & $98.97 \pm 1.16$ \\
Before discharge & $98.78 \pm 1.36$ & $99.17 \pm 1.78$ & $100.9 \pm 1.21^{\mathbf{\Delta}}$ \\
6 months after discharge & $100.58 \pm 1.33^{\mathbf{\Delta}}$ & $99.76 \pm 1.68$ & $98.62 \pm 2.57$ \\
Control group $(\mathrm{n}=71)$ & & & $99.66 \pm 2.54$ \\
Before discharge & $98.63 \pm 2.26$ & $100.12 \pm 2.76$ & $100.2 \pm 2.27^{\mathbf{\Delta}}$ \\
6 months after discharge & $100.94 \pm 2.68^{\mathbf{\Delta}}$ & & \\
\hline
\end{tabular}

$\Delta$, compared with that before treatment, $\mathrm{P}<0.05$. EP, epilepsy; SD, standard deviation; VIQ, verbal intelligence quotient; PIQ, performance intelligence quotient; FIQ, full intelligence quotient.

grouping $(\mathrm{P}<0.05$; Table 6).

\section{Discussion}

$\mathrm{EP}$ is a chronic disease. Active and regular anti-EP treatment is the basis for children with EP to control seizures and improve their quality of life. By adhering to regular and systematic medication, EP of between $80 \%$ and $90 \%$ of children can be completely controlled and these children can live, study, and work like normal 
Table 4 Quality of life of children with EP $($ mean \pm SD)

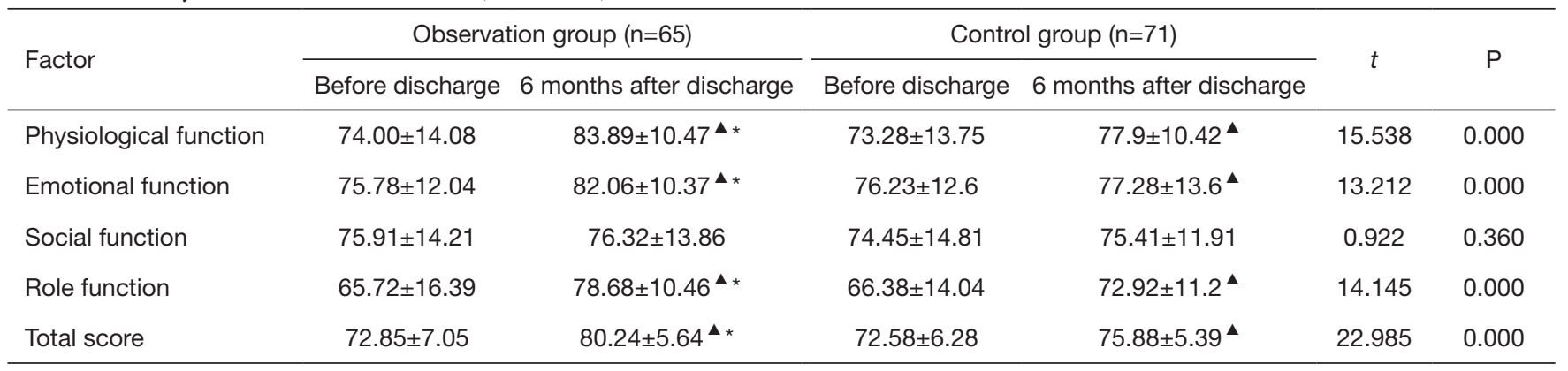

$\triangle$, compared with that before treatment, $\mathrm{P}<0.05$; ${ }^{\star}$, compared with the control group, $\mathrm{P}<0.05$. EP, epilepsy; SD, standard deviation.

Table 5 Single factor analysis of quality of life (mean \pm SD)

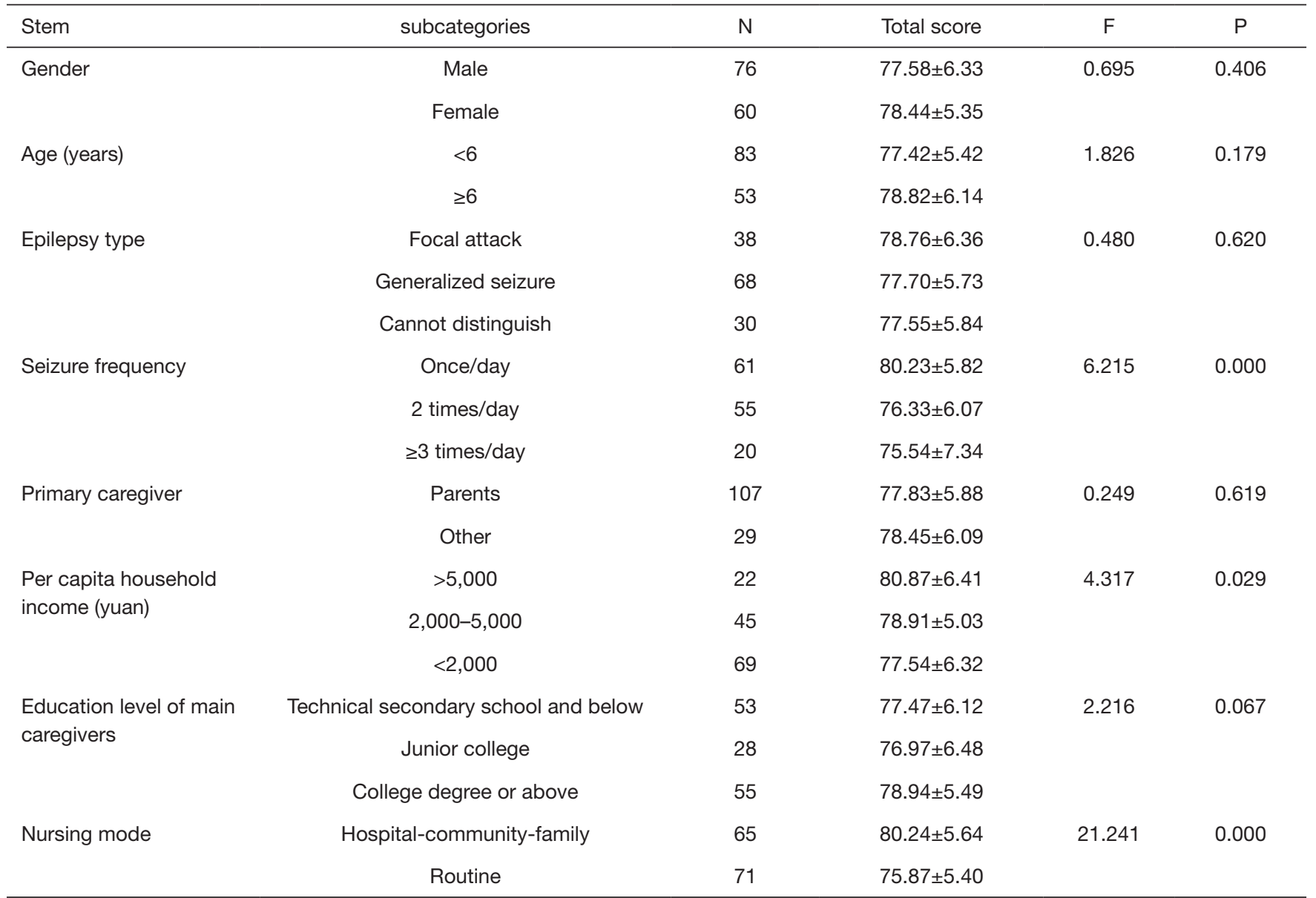

SD, standard deviation.

people (17). Health education can help children and their families realize that EP is not terrible and is controllable, change the social prejudice against EP disease, enhance the confidence of children with EP and their families, improve children's health maintenance and health management, improve children's compliance with treatment, and improve children's quality of life. The World Health Organization (WHO) definition of quality of life refers to an individual's 
Table 6 Results of multivariate regression analysis of children with EP

\begin{tabular}{lccccc}
\hline Related factor & B & SE & $\beta$ & $t$ & $P$ \\
\hline Seizure frequency & 1.764 & 0.315 & 0.591 & 6.895 & 0.000 \\
Per capita household income & 0.476 & 0.156 & 0.266 & 2.687 & 0.002 \\
Nursing mode & 2.048 & 1.360 & 2.269 & 12.763 & 0.000 \\
\hline
\end{tabular}

EP, epilepsy; SE, standard error.

perception of self-state living in a specific cultural environment and value system, which is related to his goals, expectations, standards, and social relations. Quality of life should include at least three aspects: physiology, psychology, and society (18). Research shows that the targeted education and training that EP specialist nurses carry out for patients and their families not only helps patients with EP them comply with drug management, but also reduces the number of emergency admissions, saves medical expenses, and promotes the recovery of the patients' self-esteem and social function (19). However, the cost-benefit model introduced by some health service institutions limits the development of EP specialist nurses (20). The importance of long-term management of children with EP has been an increasing concern for scholars. A key issue is that the uneven distribution of medical resources can impact on how effectively we can the existing resources to provide continuous, high-quality, and evidence-based nursing services for children and meet the needs of children and caregivers. Examining this issue in more detail is worthy of further research and discussion.

Continuing care originated from the American Hospital Association (AHA) and extended from the concept of discharge planning. The American Geriatric Society defines continuing care as designing a series of nursing activities to ensure the coordination and continuity of health services received by patients when transferring between different health care places or between health care institutions at different levels, so as to prevent or reduce the deterioration of the health status of high-risk patients (21). It generally includes the continuation of information, medical care services, and the doctor-patient relationship (22). Continuing care includes the hospital's discharge plan, referral, continuous follow-up, and guidance after the patient returns to their family or community. It also uses information tools to establish purposeful interactions between nurses, patients, and family members by means of telephone, letter, e-mail, and family visits to promote and maintain the health of patients. Extending hospital care in this way can improve patient outcomes and save human resources.

On the basis of learning from foreign experience, China's continuing care model has also made great progress. Increasing numbers of medical institutions are paying attention to the status of a patient's community and family, especially the uneven distribution of medical resources in China and the limitation of medical resources in tertiary hospitals. More scholars have expressed a vision for a hospital-community-family as a touchstone to explore the future of China's continuous nursing model. This ternary linkage form of the hospital-community-family can apply a continuing care information system to improve the cooperation and coordination ability between the hospital, community, and families. This is ultimately conducive to building trust, improving communication between nurses and patients, and maintaining continuity of care (23). Shi et al. (24) implemented hospital-community-family linkage continuous nursing model for diabetic patients, and found that patients' sense of trust was enhanced, and the function of community health service centers was highlighted. Yang et al. (25) found that with the help of hospital-communityfamily linkage continuous nursing model, in 5 months, 55 cases of diabetes patients self-management compliance rate raised from $50.9 \%$ to $89.1 \%$. Zhang et al. (26) realized the long-term effective management of stroke patients through hospital-community-family linkage continuous nursing model, and improved the doctors' work efficiency and medical service quality.

This study found that the hospital-community-family ternary linkage continuous nursing model can improve treatment compliance, children's resilience, and quality of life. Stepwise regression analysis showed that EP attack frequency, family per capita income, and the nursing model were the main factors affecting the quality of life of children with EP. The quality of life of most children improved significantly after effectively controlling the onset of EP (27), indicating that the frequency of attack is an important factor affecting the quality of life of children, which is consistent with the results of this study. 
In addition, disease attack also affects children's emotional and social functions; Völkl-Kernstock et al. (28) found that EP impairs cognitive function, which makes children's performance in learning, daily life, and communication generally lower than normal children and bears a huge psychological burden that may eventually result in various mental and behavioral problems. Kimiskidis et al. (29) found that seizure frequency is an independent risk factor for predicting depression. These further show that EP attack is an important factor affecting children's quality of life. The implementation of continuous nursing should aim to control of EP attacks as the basis for care.

In the process of implementation, the members of the ternary linkage continuous nursing model group need to go to the community hospital for relevant training and assessment, which will lead to the tension of the personnel in the department; after the intervention, some patients still had problems such as poor compliance and noncooperation. We actively found countermeasures to solve the problems, such as: planned shift arrangement for team members, one doctor and one nurse went to the community clinic in turn for one month; hospital and community health care members communicate with patients by telephone every month, establish trust relationship, check and guide regularly, and improve patients' compliance.

There were some imitations in this study: (I) the subjects of this study are all from one city, and there may be some regional bias; (II) the object of this study is limited to one hospital, which is not representative, so multi-center experimental research should be carried out; (III) due to the limitations of manpower, time, and funds, we only evaluated the follow-up nursing of children for 6 months and were unable to carry out a continuous long-term effect investigation, therefore the nursing time needs to be extended in the future.

\section{Conclusions}

The adoption of a hospital-community-family linkage continuous nursing model can improve the compliance, resilience, and quality of life of children with EP. It can be used as a new method for long-term management of children with EP.

\section{Acknowledgments}

Funding: None.

\section{Footnote}

Reporting Checklist: The authors have completed the STROBE reporting checklist. Available at https:// tp.amegroups.com/article/view/10.21037/tp-22-21/rc

Data Sharing Statement: Available at https://tp.amegroups. com/article/view/10.21037/tp-22-21/dss

Conflicts of Interest: All authors have completed the ICMJE uniform disclosure form (available at https://tp.amegroups. com/article/view/10.21037/tp-22-21/coif). The authors have no conflicts of interest to declare.

Ethical Statement: The authors are accountable for all aspects of the work in ensuring that questions related to the accuracy or integrity of any part of the work are appropriately investigated and resolved. The study was conducted in accordance with the Declaration of Helsinki (as revised in 2013). The study was approved by the Ethics Committee of Wuxi Children's Hospital (No. 2020451). Informed consent was obtained from all patients' guardians.

Open Access Statement: This is an Open Access article distributed in accordance with the Creative Commons Attribution-NonCommercial-NoDerivs 4.0 International License (CC BY-NC-ND 4.0), which permits the noncommercial replication and distribution of the article with the strict proviso that no changes or edits are made and the original work is properly cited (including links to both the formal publication through the relevant DOI and the license). See: https://creativecommons.org/licenses/by-nc-nd/4.0/.

\section{References}

1. Fiest KM, Sauro KM, Wiebe S, et al. Prevalence and incidence of epilepsy: A systematic review and meta-analysis of international studies. Neurology 2017;88:296-303.

2. Subspecialty Group of Neurology, the Society of Pediatrics, Chinese Medical Association. Expert consensus on long-term management of childhood epilepsy. Chinese Journal of Pediatrics 2013;51:699-703.

3. Zhu F, Lang SY, Wang XQ, et al. Long-term Effectiveness of Antiepileptic Drug Monotherapy in Partial Epileptic Patients: A 7-year Study in an Epilepsy Center in China. Chin Med J (Engl) 2015;128:3015-22.

4. Ronen GM, Streiner DL, Verhey LH, et al. Disease 
characteristics and psychosocial factors: explaining the expression of quality of life in childhood epilepsy. Epilepsy Behav 2010;18:88-93.

5. Mott J, Shellhaas RA, Joshi SM. Knowledge of epilepsy and preferred sources of information among elementary school teachers. J Child Neurol 2013;28:740-4.

6. Li H, Zhao L, Wang L, et al. Research progress on stigma of EP patients. Chinese Nursing Management 2017;17:1278-81.

7. Miller GF, Coffield E, Leroy Z, et al. Prevalence and Costs of Five Chronic Conditions in Children. J Sch Nurs 2016;32:357-64.

8. Modi AC, Wu YP, Rausch JR, et al. Antiepileptic drug nonadherence predicts pediatric epilepsy seizure outcomes. Neurology 2014;83:2085-90.

9. Michaelis R, Tang V, Goldstein LH, et al. Psychological treatments for adults and children with epilepsy: Evidencebased recommendations by the International League Against Epilepsy Psychology Task Force. Epilepsia 2018;59:1282-302.

10. Modi AC, Rausch JR, Glauser TA. Patterns of nonadherence to antiepileptic drug therapy in children with newly diagnosed epilepsy. JAMA 2011;305:1669-76.

11. El-Radhi AS. Management of seizures in children. Br J Nurs 2015;24:152-5.

12. Fisher RS, Acevedo C, Arzimanoglou A, et al. ILAE official report: a practical clinical definition of epilepsy. Epilepsia 2014;55:475-82.

13. Morisky DE, Green LW, Levine DM. Concurrent and predictive validity of a self-reported measure of medication adherence. Med Care 1986;24:67-74.

14. Zhang H. Revision of Chinese version of Wechsler Intelligence Scale for children (WISC-IV). Journal of Psychological Science 2009;32:1177-9.

15. Kim DH, Yoo IY. Development of a questionnaire to measure resilience in children with chronic diseases. J Korean Acad Nurs 2010;40:236-46.

16. Lu Y, Tian Q, Hao Y, et al. Reliability and validity analysis of Chinese version of child quality of life scale PedsQL4.0. Journal of Sun Yat-sen University (Medical Sciences) 2008;131:328-31.

17. Bozkaya IO, Arhan E, Serdaroglu A, et al. Knowledge of, perception of, and attitudes toward epilepsy of schoolchildren in Ankara and the effect of an educational program. Epilepsy Behav 2010;17:56-63.

18. Mwanyangala MA, Mayombana C, Urassa H, et al. Health status and quality of life among older adults in rural Tanzania. Glob Health Action 2010.

19. Pfäfflin M, Schmitz B, May TW. Efficacy of the epilepsy nurse: Results of a randomized controlled study. Epilepsia 2016;57:1190-8.

20. Hopkins J, Irvine F. Qualitative insights into the role and practice of Epilepsy Specialist Nurses in England: a focus group study. J Adv Nurs 2012;68:2443-53.

21. Shortell SM, Rundall TG, Hsu J. Improving patient care by linking evidence-based medicine and evidence-based management. JAMA 2007;298:673-6.

22. Saultz JW, Albedaiwi W. Interpersonal continuity of care and patient satisfaction: a critical review. Ann Fam Med 2004;2:445-51.

23. Cossette S, Frasure-Smith N, Vadeboncoeur A, et al. The impact of an emergency department nursing intervention on continuity of care, self-care capacities and psychological symptoms: secondary outcomes of a randomized controlled trial. Int J Nurs Stud 2015;52:666-76.

24. Shi Y, Wang X, Sun X. The application of ternary linkage health care model in continuing nursing care of diabetic patients. Chinese Nursing Management 2012;12:8-11.

25. Yang H, Wang P, Hou W, et al. Design and application of hospital community family ternary linkage continuing care platform. Chinese Journal of Nursing 2016;51:1133-7.

26. Zhang S, Han X, Wu D, et al. Establishment of a new regional management model of stroke based on the smart medical consortium platform. Fudan University Journal of Medical Sciences 2018;45:805-10.

27. Bhattacharya R, Chakraborty S, Sanyal D, et al. An Unique Case of Ito Syndrome Presenting with Juvenile Myoclonic Epilepsy and Normal Intelligence. International Journal of Psychology and Psychiatry 2014;2:238-46.

28. Völkl-Kernstock S, Bauch-Prater S, Ponocny-Seliger E, et al. Speech and school performance in children with benign partial epilepsy with centro-temporal spikes (BCECTS). Seizure 2009;18:320-6.

29. Kimiskidis VK, Triantafyllou NI, Kararizou E, et al. Depression and anxiety in epilepsy: the association with demographic and seizure-related variables. Ann Gen Psychiatry 2007;6:28.

(English Language Editor: C. Mullens)

Cite this article as: Gao Y, Tang X, Wen Y, Qian D, Pan X, Zhang L. Effects of the hospital-community-family ternary linkage continuous nursing model on compliance, cognitive function, resilience, and quality of life for children with epilepsy: a retrospective study. Transl Pediatr 2022;11(2):239-248. doi: $10.21037 /$ tp-22-21 